\title{
Simulation of Allocation of Reliability in Component based System
}

\author{
Karambir \\ Assistant Professor, Department of CSE \\ University Institute of Engineering and Technology \\ Kurukshetra University, Kurukshetra, Haryana
}

\author{
P. K. Suri \\ Dean(Academic and R\&D) \\ Chairman \& Professor(CSE/IT/MCA) \\ HCTM Technical Campus, Kaithal, Haryana
}

\begin{abstract}
The reliability allocation and minimum cost of component in a system can be used to solve the allocation problems in components based systems as well as applicable to modular systems. A method is simulated for allocating reliability to each component of a system with a view to minimizing the system cost on specific reliability. There is a need for component developer and users to make these data available to reliability estimator so that the derived results are usefully applied to practical systems. The simulation to show near optimal solution to the problem of selecting the component and comprising the software can be obtained with minimum cost. We simulate different sizes of components and their reliability with minimum cost in a system. Finally we give the importance of how to select reliability and cost of each component in Component-based software.
\end{abstract}

\section{Keywords}

Component Based System, Simulation Software, Software Reliability, Reliability Allocation, Reliability Estimation

\section{INTRODUCTION}

Software system is used in everywhere on our daily lives. Suppose a failure can be occurred during the operation of software can lead to economic loss and waste of time and may even cause loss of human lives also. If the unreliable software is identified in the early stage, loss may be small. Therefore unreliable software is not acceptable and it should be identified in the beginning of the software development. If it is not identified initial stage and later the defects found, the higher the cost that needs to paid for them. Software Reliability is one of the very important and quality attributes of software since it quantifies software failures during the development process. If the higher level of Reliability of software can definitely result in more development cost. In practical, software project managers are required to optimize the cost needed to complete the development of software system on some specific value of reliability. So many software reliability models had been proposed in the past decades to help the software managers and developers to analyze and design the system. The main constraint in software development is maximizing reliability and minimizing cost.

Reliability allocation is concerned with the setting of reliability goals for individual component such that a specified reliability goal[1] is met and the reliability of all the components are well balanced so that the minimization of overall development cost. Reliability allocations [2] for components based systems can be started as soon as the system reliability models have been adopted for developing and selecting the components. The initial values allocated to the system itself should either be the specified values for the various reliability metrics for the system, or a set of reliability values which are marginally more difficult to achieve than the specified values. Reliability values that are maximum that are allocated to the system to allow for later system functionality growth and to allow those parts of the system which cannot achieve their allocated values. The apportionment of reliability values between the various subsystems and elements can be made on the basis of complexity, criticality, estimated achievable reliability, or any other factors considered appropriate by the analyst making the allocation[3]. The procedures provided for allocation of software failure rates can be applied to all the components that these are working in series or concurrently. System-level allocations are successively decomposed using the reliability models until an appropriate set of reliability measures has been apportioned to each component of the system.

In this paper we simulated the integration of component by assuming initial reliability in a small and large case software. In testing phase we have many constraints like when to stop testing and limit of cost on testing. First one is number of components and second one is design. The components may be depends on the size of the project structure. This paper takes the following arrangement. Section 2 introduces software reliability allocation model. Section 3 depicts how to find out the optimal allocation method by using a dynamic programming algorithm. Section 4 illustrates the application of the algorithm proposed in section 3, section 5 shows the simulation result and its analysis. Section 6 offers concluding remarks and directions .

\section{SOFTWARE RELIABILITY ALLOCATION MODEL}

The cost of developing any software is inversely proportional to the reliability metrics of that software. The pretended software development cost minimization model[4] can be proposed having two directions. One is to apply an reliability allocation method while achieving the goal reliability such that the delivery cost of that software can be as small as possible; the next is how much to allocate the reliability to individual component so that the system reliability can be maximized on the condition of that there is no compromise to the quality of system. Components based systems are executed by using a set of interrelated subsystems in a series pattern by message passing or calling the other component. Reliability allocation means the fixing of the reliability to components so that cost can be optimized.. Generally there are number of the allocation algorithm is used to deal with the optimal solution with some constraints. 


\section{SOFTWARE RELIABILITY COST MODEL}

\subsection{Assumptions}

Please The assumptions for developing the Software reliability and cost model are (1) the cost of system is the sum of cost of individual component.(2) the reliability of system is equal to the multiplication of reliability of active components. (3) high reliability can achieved by good design, skilled worker and giving more time in testing phase. as a result of this, the development cost of that system increases.(4) the reliability can not be $100 \%$ for any software component/ system. The fourth condition suggests that it can be very expensive to achieve the reliability value of $100 \%$. In fact for software, it has been shown that under some assumptions, it is infeasible to achieve ultra-high reliability in software[3].

The cost function of each component would satisfy these three conditions(1) it should have positive value (2) it should be increasing with time (3) high value for high reliability. In some cases, the cost function can be derived from basic considerations and is usually stated in terms of the reliability, as we will do below for software reliability. According to the definition of software reliability [9].

$$
R(t)=e^{-t * \lambda}----(1)
$$

Where $\mathrm{R}(\mathrm{t})$ is continuous-time system reliability, and $\lambda$ is its failure rate.From the above equation, it can be interpreted that the reliability of a software is directly dependent on the number of faults that remain in it after testing. The system failure rate is proportional to the number of faults. To remove more fault of the system, advanced software, skilled tester are required and as a result the cost is increased. Software development cost is proportional to the complexity and size of the software system.

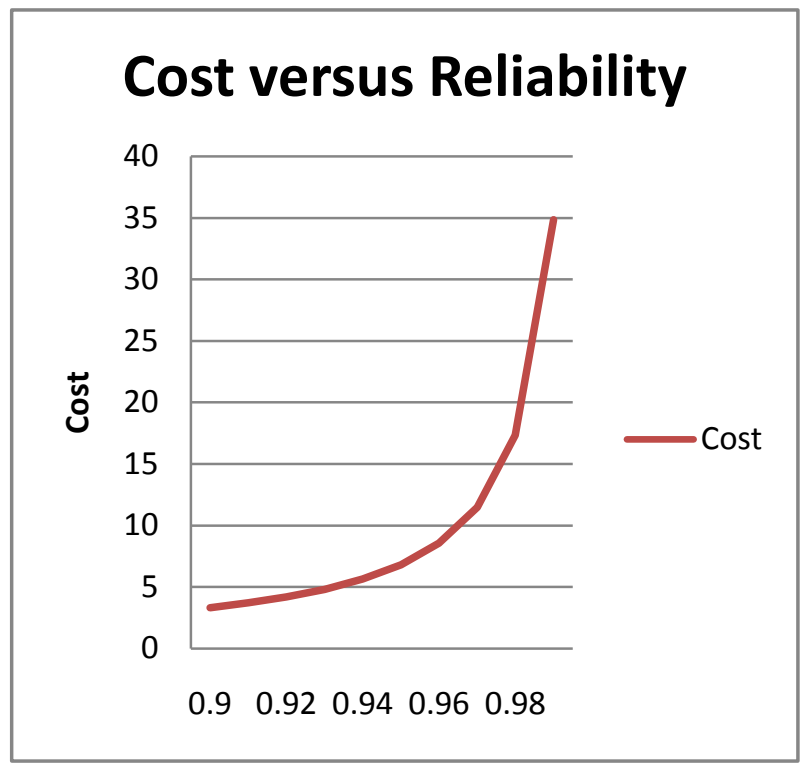

Fig 1: Cost versus Reliability

Where $\mathrm{k}$ represents the complexity and size for developing the software system and is called reliability-cost coefficient and some basic cost such as personnel training, development tools preparation etc in this paper.

\subsection{Software Reliability Allocation Model}

Here we assume the software system has been designed as an assembly of appropriated connected components. Let there be $n$ components, each with reliability $\operatorname{Ri}(t)$ and cost Ci [5]for i=1...n.. Let R(target_system) be the specified target reliability and $\mathrm{R}$ and $\mathrm{C}$ represent the overall reliability and the total system cost. Let

$$
\begin{aligned}
& F(R) \\
& =\prod_{i=1}^{n} R_{i} \geq R\left(\text { target }_{\text {system }}\right)----(2)
\end{aligned}
$$

The software reliability allocation model can be stated as: Objective function to be minimum

$$
C=\sum_{i=1}^{n} C_{i}----(3)
$$

development cost and the system reliability shall be no less than 0.85. the term and condition for allocating the reliability to each component. Set the precision of computing is 0.01 .

Such a problem can be defined as:

$$
R(\text { total })=\prod_{i=1}^{6} R_{i} \geq 0.85----(4)
$$

This algorithm try to reallocate the reliability of each subsystem or components so as to achieve the target reliability while minimizing the total development cost.

As for a software system with $\mathrm{n}$ subsystems or components within a given architecture, the development cost of the system will increase while enhancing the software reliability target. Significantly, the greater improvement of the reliability, the more increase of the cost will be required.

\section{ALGORITHM TO SOLVE RELIABILITY ALLOCATION PROBLEM}

\subsection{Algorithm}

A software system with $\mathrm{n}$ components and the association function $\mathrm{F}$ discussed above is known. The reliability-cost coefficient $\alpha$ of each component and the specified system reliability target $\mathrm{R}$ obj is given.

Step 1: set $R=[0.99,0.99,0.99,0.99,0.99,0.99]$ and costreliability coefficient $\mathrm{k}=[-0.35-0.43-0.51-0.65-0.74$ -

$0.91]$;

// the reliability matrix $[\mathrm{r} 1, \mathrm{r} 2, ., \mathrm{rn}]$ and $\mathrm{C}$ represent the cost matrix $[\mathrm{c} 1, \mathrm{c} 2, \ldots, \mathrm{cn}]$,

Step 2: Repeat loop $i:=1$ to 40 times [begin]

for $\mathrm{j}:=1: 40$

Step 3: set $\mathrm{z}:=1$;

Step 4: Repeat $y=1$ to6 times[begin]

Step 5: set $\quad \mathrm{z}=\mathrm{z} * \mathrm{R}(\mathrm{y})$; [end loop]

Step 6: if $z>=0.85$ [begin] //required reliability of system 
Step 7: repeat $i:=1$ to 6 times [begin]

Step 8: temp:=R //copy reliability matrix to temp matrix

Step 9: repeat $\mathrm{x}:=1$ to 6 times[begin]

Step 10: if $x=1$ then [begin]

Set temp $(x)=\operatorname{temp}(x)-0.01$;

Else

Set temp $(\mathrm{x})=\operatorname{temp}(\mathrm{x}) ;$ [endif] [endloop]

set $\mathrm{t} \_$cost $=0$;

Step 11: repeat loop for $c:=1$ to 6 time[begin]

Set t_cost:=t_cost+k(c)/log(b(c)); [endloop]

Step 12: set cost $(\mathrm{i})=\mathrm{t} \_$cost; [endloop]

Step 13: set minimum $\mathrm{m}:=\min (\operatorname{cost})$; $\operatorname{disp}(\operatorname{cost})$

Step 14: repeat loop for $t:=1$ to 6 [begin]

Step 15: if $m=\cos t(t)$

Set $\quad \mathrm{R}(\mathrm{t}):=\mathrm{R}(\mathrm{t})-0.01$;

Else Set $\mathrm{R}(\mathrm{t}):=\mathrm{R}(\mathrm{t})$; [endif][endloop]

Else break; [endif][endfor]

Step 16: [end of program]

Where $R(t)$ is the result of reliability allocation[7] of each component after simulation. $\mathrm{R}$ matrix and Cost matrix are the corresponding system reliability and expected system development cost. K matrix is the expected development cost coefficient allocated to each component. Noted from the above that term and minimum condition to the implementation of the algorithm is that the decrease in reliability of one component can result in that of the whole system and lower the development cost. But that can be guaranteed in our algorithm. The aim of algorithm is to select an optimal component whose decrease in reliability can result in the maximal cost versus reliability variation, which makes the single step programming optimized so that optimal reliability allocation of the ultimate system is guaranteed.

\section{RESULT AND SIMULATION}

The Here we design a system with six independent components having $\mathrm{r} 1, \mathrm{r} 2, \mathrm{r} 3, \mathrm{r} 4, \mathrm{r} 5$ and $\mathrm{r} 6$. We assume that all the components are active while executing the system and their failures are statistically independent and exponential distributed. Therefore, the relationship between the total system reliability $r$ and its components' reliability $\mathrm{R}(\mathrm{i})(\mathrm{i}=1: 6)$ can be stated as: $\mathrm{r}=\mathrm{F}(\mathrm{r} 1, \mathrm{r} 2, \mathrm{r} 3, \mathrm{r} 4, \mathrm{r} 5, \mathrm{r} 6)=$ $\mathrm{r} 1 *_{\mathrm{r} 2} *_{\mathrm{r}} 3 *_{\mathrm{r}} 4 *_{\mathrm{r}} 5 *_{\mathrm{r}} 6$. Suppose that the reliability cost coefficient of the components [6] are 0.35, 0.43, 0.51, $0.65,0.74$ and 0.91 respectively. In order to minimize the system development cost and the system reliability shall be no less than 0.85 . the term and condition for allocating the reliability to each component. Set the precision of computing is 0.01 .

The cost of component can be assumed as by the following expression
(1) $\mathrm{c} 1=-0.35 / \mathrm{ln} \mathrm{r} 1$
$\mathrm{c} 2=-0.43 / \ln \mathrm{r} 2$
(2) $\mathrm{c} 3=-0.51 / \mathrm{ln} \mathrm{r} 3$
$\mathrm{c} 4=-0.65 / \ln \mathrm{r} 4$
(3) $\mathrm{c} 5=-0.74 / \ln \mathrm{r} 5$
(6)
$\mathrm{c} 6=-0.91 / \mathrm{ln} \mathrm{r} 6$

Estimate the values of reliability parameters of different component $(\mathrm{r} 1, \mathrm{r} 2, \mathrm{r} 3, \mathrm{r} 4, \mathrm{r} 5, \mathrm{r} 6)$ with which the total cost $\mathrm{C}$ $(\mathrm{C}=\mathrm{c} 1+\mathrm{c} 2+\mathrm{c} 3+\mathrm{c} 4+\mathrm{c} 5+\mathrm{c} 6)$ is minimized. With respect to each component, we compute the cost with the reliability from 0.91 to 0.99 (increment is 0.01 ) according to the reliability-cost model.

According to the algorithm above, set initial state R0 = $[0.99,0.99,0.99,0.990 .990 .99]$. As per equation the cost of components are, $\mathrm{C} 0=\left[\begin{array}{lll}34.82 & 42.7850 .74 & 64.67\end{array}\right.$ $73.6390 .54], \delta=0.01$, and the system cost will be

$\mathrm{C} 0=357.20$, system reliability $\mathrm{R} 0=0.99$ * $0.99 * 0.99 * 99 * 0.99 * 0.99=0.94$.

\section{total cost of system}

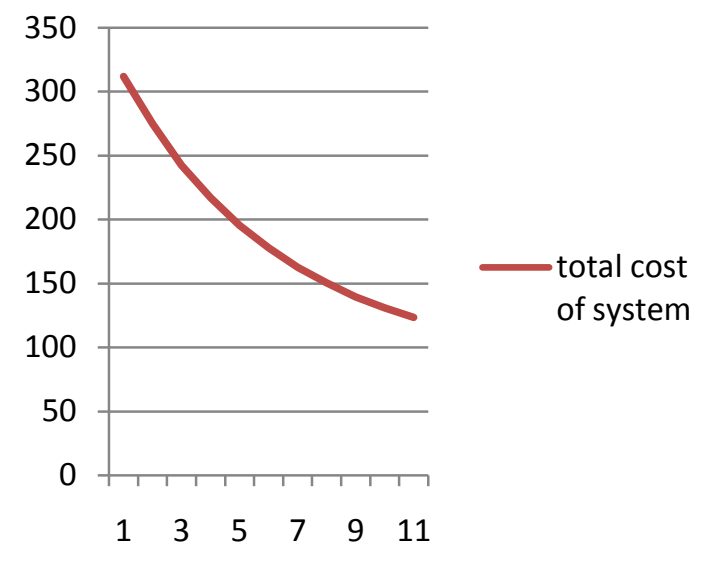

Fig2: Total Cost of System

Set $\mathrm{i}=1,2,3,4,5$ and 6 then compute separately with different value. Fig 2 shows that the cost of system increases as the reliability increases. In fig 3 , the effect of reliability of different component has represented and it shows that if the reliability of particular component is focused then the total cost of system may not be optimized.

Table 1: Cost Effectiveness of Component Replacement

\begin{tabular}{|r|r|r|r|}
\hline Iteration & \multicolumn{1}{|l|}{$\begin{array}{l}\text { System } \\
\text { cost }\end{array}$} & $\begin{array}{l}\text { Cost change } \\
\text { due } \\
\text { component-1 }\end{array}$ & $\begin{array}{l}\text { Cost change } \\
\text { due } \\
\text { component-2 }\end{array}$ \\
\hline 1 & 311.7 & 339.7 & 335.7 \\
\hline 2 & 274.7 & 294.2 & 290.2 \\
\hline 3 & 242.2 & 257.2 & 253.2 \\
\hline 4 & 216.7 & 224.7 & 220.7 \\
\hline 5 & 195.2 & 199.2 & 195.2 \\
\hline 6 & 177.7 & 177.2 & 18.03 \\
\hline 7 & 162.53 & 171.87 & 170.53 \\
\hline 8 & 150.2 & 156.7 & 155.36 \\
\hline 9 & 139.36 & 144.36 & 143.06 \\
\hline 10 & 130.86 & 133.53 & 132.2 \\
\hline 11 & 123.28 & 125.03 & 123.7 \\
\hline
\end{tabular}




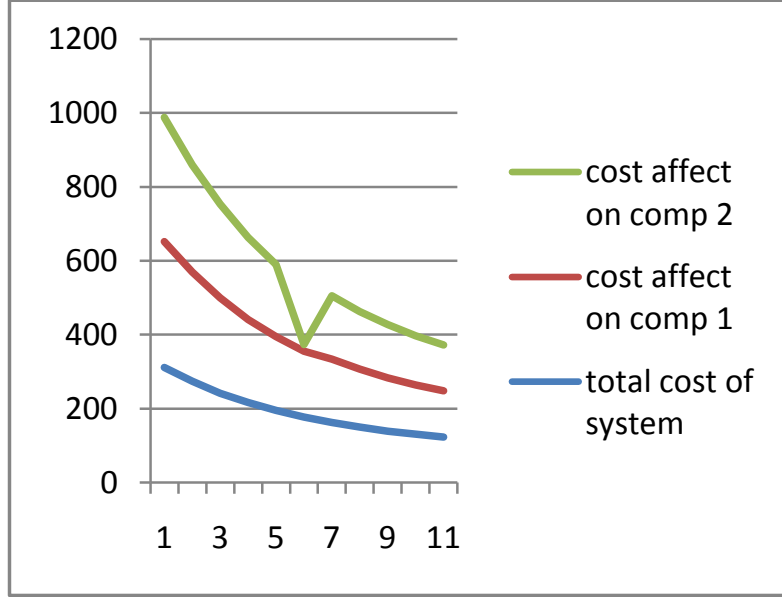

Fig3: effect of component on total cost of system

\section{CONCLUSION}

Software reliability allocation for Component based system shows an important role during software product design phase and phase which is a optimization of cost with software modeling. We formulated an reliability cost model for software reliability optimization solution, on this basis a dynamic programming algorithm has been proposed and analyzed the simulated result in this paper which can be used to allocate the reliability to each component so as to minimize the cost of designing software while meeting the desired reliability objective. The result of our simulation show an optimal or approximate optimal solution to the problem of selecting the various having different reliability value of components comprising software. The reliability and cost allocation model presented in this paper can be used to solve the optimal allocation problems in simple systems. It can be concluded from the result the particular time should be given to a particular component of the system so that the reliability of system is sufficient enough for designing a system and saving the extra cost of developer.

\section{REFERENCES}

[1] Aggarwal, K.K., "Minimum Cost Systems with Specified Reliability", IEEE Transactions on Reliability ,Volume:R-26, Issue: 3, pp 166-167, 2009

[2] Srinivasarao.Sabbineni and Kurra Rajasekhararao, "Estimation of Reliability Allocation on Components Using a Dynamic Programming", IJCSI International Journal of Computer Science Issues, Vol. 10, Issue 3, No 1, May 2013

[3] R. W. Bulter and G.B. Finelli, "The infeasibility of quantifying the reliability of life-critical real-time software", IEEE Transactions on Software Engineering ,pp 19:3-12,, 1993

[4] M.E. Helander, M. Zhao and N. Ohlsson, "Planning Models for Software Reliability and Cost". IEEE Trans. on Software Engineering, 24(6), pp 420-434, 1998,

[5] Hui Guan, Wei-Ru Chen, Ning Huang andHong-Ji Yang, "Estimation of Reliability and Cost Relationship for Architecture-based Software", International Journal of Automation and Computing 7(4), pp 603-610, November 2010.

[6] F. Zahedi and N. Ashrafi, "Software Reliability Allocation Based on Structure, Utility, Price and Cost", IEEE Transaction on Software Engineering, 17 (4), pp 345-356, 1991

[7] M. R. Lyu. Handbook of Software Reliability Engineering. IEEE Computer Society Press, New York, pp.36, 1996

[8] Srinivasarao.Sabbineni and Kurra.Rajasekhara, "Assessment of Architecture based Software System Reliability Allocation on Components using a Dynamic Programming", International Journal of Computer Applications, Volume 73,No.19, July 2013.

[9] P. K. Suri and Karambir," Siumlation of reliability of Software Component", Global Journal of Computer Science and Technology, volume-13, issue -13 , pp$13,17,2013$ 